\title{
Effectiveness of Ranolazine to Prevent Myocardial Injury During Elective Percutaneous Coronary Intervention
}

\author{
*SMM Iqbal ${ }^{1}$, SA Ahsan ${ }^{2}, \mathrm{KA} \mathrm{Jahan}{ }^{3}, \mathrm{SN} \mathrm{Eva}^{4}$
}

\begin{abstract}
Background: Ranolazine is a novel antianginal drug that reduces intracellular accumulation of calcium ion in ischemic myocardium. A pilot randomized study $(n=70)$ has shown that pretreatment with ranolazine $1000 \mathrm{mg}$ twice daily for 7days significantly reduced periprocedural myocardial injury (PMI) in elective Percutaneous coronary intervention (PCI). Our objective was to detect whether similar effect could be obtained by ranolazine pretreatment through an interventional study.

Materials \& Methods: 110 patients with chronic stable angina scheduled for elective PCI were enrolled in an interventional study. For 7 days before the procedure, 55 patients were allocated to receive ranolazine $1000 \mathrm{mg}$ twice daily (ranolazine group) and 55 patients didn't receive ranolazine (control group). Serum creatinine kinase-MB (CK-MB) and Troponin I levels were measured at baseline and 24 hours post procedure.
\end{abstract}

Results: Periprocedural myocardial injury [i.e. an elevation of serum biomarkers (preferably cardiac troponins) above the $99^{\text {th }}$ percentile of upper reference limit (URL)] was detected less commonly after PCI in ranolazine than in control group $(11 \%$ vs. $27 \%, \mathrm{p}=0.0001)$. Also, PCI-related myocardial infarction [i.e., post procedural increase in CK-MB $>3$ times above the URL] tended to be lower in the ranolazine versus placebo group: $1.8 \%$ versus $5.45 \%, \mathrm{P}=0.0002 .24$ hours post procedural levels of cardiac markers were also significantly lower in the ranolazine versus control group (CK-MB: $2.42 \pm 2.05$ versus $7.02 \pm 9$ $\mathrm{ng} / \mathrm{ml}, \mathrm{P}=0.001$; Troponin 1: $0.447 \pm 0.74$ versus $1.18 \pm 1.6 \mathrm{ng} / \mathrm{ml}, \mathrm{P}=0.004)$. No significant adverse effect of the drug was reported.

Conclusion: So, we have concluded that ranolazine was effective in significantly reducing the periprocedural myocardial injury in elective PCI.

Key Words: Ranolazine, Periprocedural Myocardial Injury, Elective Percutaneous Coronary Intervention.

\section{Introduction}

Coronary artery disease (CAD) is now the most common cause of death worldwide; it is on the rise and has become a true pandemic that respects no borders. ${ }^{1}$ According to the Global Burden of Disease Study ${ }^{2}$, the developing countries contributed 3.5 million of the 6.2 million global deaths for CAD in 1990. The projections estimate that these countries will account for 7.8 million of the 11.1 million deaths due to CAD in 2020. In 2005, CAD caused approximately 1 of every 5 deaths in the USA. $^{3}$

Bangladeshi people, like other south Asians, have high susceptibility to ischemic heart disease (IHD) but population-based data are lacking in Bangladesh.

\footnotetext{
$1 *$ Dr. S M Mamun Iqbal, Senior Consultant, Cardiology, AFC Health Fortis Heart Institute, Cumilla e-mail: kironk56@yahoo.com

${ }^{2}$ Prof. Syed Ali Ahsan, Professor, Cardiology, Bangabondhu Sheikh Mujib Medical University, Shahbag, Dhaka

${ }^{3}$ Dr. Kasekh Akhtar Jahan, Manager, Quality Control, AFC Health Fortis Heart Institute, Khulna

${ }^{4}$ Dr. Sohely Nazneen Eva, Specialist, Laboratory Medicine, United Hospitals limited, Dhaka

*Corresponding Author

Date of submission: 04.08.2018 Date of acceptance: 11.11.2018
}

AKMMC J 2019; 10(1) : 43-49 
A prevalence of $3.4 \%$ was recorded in rural population with traditional lifestyle and thin body mass index. ${ }^{4}$

Bhopal $\mathrm{R}$ et al in a cross sectional study 5 among the south Asian people in United Kingdom showed that for most risk factors, the Bangladeshis (particularly men) fared the worst: smoking was most common $(57 \%)$ in them and Bangladeshis had the highest concentrations of triglycerides $(2.04 \mathrm{mmol} / \mathrm{L})$ and fasting blood glucose $(6.6 \mathrm{mmol} / \mathrm{L})$ and the lowest concentration of high density lipoprotein cholesterol (0.97 mmol/L).

The treatment of CAD has evolved considerably over the past decades. Along with lifestyle modification and pharmacological treatment, percutaneous coronary intervention (PCI) has become a standard revascularization procedure for patients with CAD. Since the first description of coronary angioplasty in man by Andreas Gruntzig in 1977, the procedure has been extensively modified ${ }^{6}$. About one-third of all elective PCI procedures are associated with significant myocardial injury, termed peri-procedural myocardial injury (PMI), which has been associated with increased subsequent mortality ${ }^{7}$.

The most common (50-75\%) mechanisms of myocardial injury during PCI are distal embolization and side branch occlusion $(\mathrm{SBO})^{6}$. Other significant causes include dissection, thrombus, no reflow or slow flow; or coronary perforation ${ }^{6}$.

Cardiac biomarkers have been extensively used in the past two decades to establish the incidence and the prognostic implication of $\mathrm{PMI}^{6}$. Several metaanalyses have inferred the proportionate increase in the early and late mortality with increased creatinine phosphokinase MB isoenzyme (CK-MB) and Troponin release periprocedurally $7,8,9,10$.

Currently, there are some pharmacologic agents to combat PMI6eg, antiplatelets, antithrombotic, statins, beta blockers, adenosine, trimetazidine etc. But PMI still remains the most frequent complication of PCI occurring in 5-30\% of patients $^{11}$. So, new adjunctive therapy is needed for better tackling PMI.
Ranolazine is a new antianginal drug that was approved on January 27, 2006 in the United States for use in patients with chronic stable angina who continue to be symptomatic on $\beta$ blockers, calcium antagonists or nitrates ${ }^{12}$. Ranolazine has the potential to partially disrupt the consequences of cell hypoxia during transient myocardial ischemia by reducing excess late sodium ion influx; thereby reducing intracellular calcium overload. The efficacy and safety of ranolazine in ischemic heart disease is well established in different randomized clinical trials. ${ }^{13,14,15,16}$

A recently completed Randomized, double-blind, placebo-controlled pilot study has shown that pretreatment with ranolazine $1000 \mathrm{mg}$ twice daily for 7 days significantly reduced procedural myocardial injury in elective $\mathrm{PCI}^{17}$. However, no such study has yet been done in Bangladesh. As the socio demographic factors, ethnicity and physical factors of patients in Bangladesh are different from the patients of European origin, we carried out such a study in our institute. In this study we have verified whether pretreatment with Ranolazine before elective PCI has any protective effect against PMI.

\section{Materials \& Methods}

110 patients with chronic stable angina scheduled for elective PCI at University Cardiac Center, BSMMU from June 2012 to February 2013, who fulfilled the eligibility criteria were enrolled in a prospective, single center, interventional study.

\section{Eligibility Criteria}

i) Inclusion criteria -

- Angiographically proven significant coronary artery disease.

- Class I indication for elective PCI,

ii) Exclusion Criteria:

- Acute coronary syndromes (unstable angina or myocardial infarction) within 3 months.

- Any increase in baseline values of markers of myocardial damage (Troponin I and creatinine kinase-MB)

- Renal failure with estimated glomerular filtration rate $(\mathrm{eGFR})<60 \mathrm{ml} / \mathrm{min} / 1.73 \mathrm{~m}^{2}$. 
- An increase in liver enzymes, especially SGPT, more than twice the upper reference limit.

- Left ventricular ejection fraction $<40 \%$.

- Prolongation of corrected QT interval in the baseline ECG $(>0.45 \mathrm{sec}$ for males $\&>0.46 \mathrm{sec}$ in females).

- Patients taking drugs known to prolong QT interval, e.g. Class Ia \& Class III anti-arrhythmic drugs, tricyclic antidepressant drugs, phenothiazines, macrolides etc ${ }^{18}$.

- Patients taking drugs that are moderately potent cytochrome P450 3A4 enzyme inhibitors ${ }^{12}$, e.g. ketoconazole, paroxetine, diltiazem, verapamil, macrolides, HIV protease inhibitors and grape fruit juice.

- Patients who had been taking ranolazine before enrollment.

- History of chronic liver disease or muscle disease.

The study conforms to the ethical guidelines of the 1975 Declaration of Helsinki and was approved by the Institutional Review Board (IRB) of our institution (Registration number: BSMMU/2012/05 87). The sample size was calculated at $5 \%$ level of significance and $90 \%$ power using a relevant formula. ${ }^{19,20}$ For 7 days before the procedure, 55 patients were allocated to receive Ranolazine 1000 mg twice daily (Ranolazine group) and 55 patients didn't receive Ranolazine (control group). The baseline characteristics of the patients of both Ranolazine and control group ( Age; Gender; BMI; Risk factors for atherosclerosis: DM, HTN, dyslipidemia, current smoking; coronary angiographic diagnosis and characteristics of coronary artery lesions) were well-balanced prior to allocation. All patients in both group were pretreated with oral aspirin $(75 \mathrm{mg} /$ day $)$ and had a loading dose of Clopidogrel $600 \mathrm{mg}$ before the procedure. Other medications such as ( $\beta$-blockers, calcium antagonists, statins and angiotensin converting enzyme (ACE) inhibitors or angiotensin receptor blockers (ARB) were given as appropriate. During enrollment, ECG was performed and blood samples were taken before PCI to analyze baseline Troponin I and CK-MB values at the same laboratory. Angiographic analysis was performed with a computer-assisted automated edge-detection system (SIEMENS AXIOM, Germany). During PCI, all patients were given a $60 \mathrm{IU} / \mathrm{kg}$ intravenous bolus of unfractionated heparin. Stents were deployed in femoral arterial route according to current practice guidelines and any complication occurring during PCI was noted. Glycoprotein IIb/IIIa inhibitors and nitrates were administered according to the operator's discretions. Blood samples were taken at 24 hours after PCI for measurement of CK-MB and Troponin I using standard laboratory method.21,22 Standard 12-lead electrocardiograms were recorded in all patients before PCI, at the end of PCI, and at 24 hours after PCI. Any symptom occurring within the first 72 hours after PCI (i.e. during hospital stay) was recorded. All patient received Clopidogrel 75 $\mathrm{mg} /$ day for at least 1 month in addition to continued aspirin $(75 \mathrm{mg} /$ day) after PCI. Data were collected using a structured questionnaire containing the key variables of interest. Variables were defined by standard operational definitions. ${ }^{2,4,23-29}$ Parametric data were compared between two groups by Student's t test and categorical data were compared between two groups by Chi Square test or Fisher's exact test. The primary end point was the occurrence of periprocedural myocardial injury (PMI) i.e., an elevation of serum biomarkers (preferably cardiac troponins) above 99 thpercentile of upper reference limit after PCI assuming a normal baseline troponin value.The secondary end point was the occurrence of PCI-related myocardial infarction i.e., an elevation of serum cardiac biomarkers to more than three times the 99th percentile of URL. The composite end point of the analysis was the incidence of Major Adverse Cardiac Events (MACE) defined as death, spontaneous myocardial infarction (MI), target vessel revascularization (TVR) or stroke, by 30 days of PCI.

\section{Results}

Among 132 patients admitted for elective PCI with a diagnosis of chronic stable angina, 110 patients were ultimately eligible for our study. The enrolled participants were allocated to Ranolazine group 
$(\mathrm{n}=55)$ and control group $(\mathrm{n}=55)$. The baseline characteristics and angiographic characteristics were well-balanced between the two groups. The procedural characteristics (use of direct stenting, maximum pressure inflation and use of post-dilatation), number of drug-eluting stents, stent diameter and the length of stents were similar in both the groups. Stents were deployed in all patients and angiographic success was achieved in all of them. The occurrence of procedural complications was similar in both the groups. Periprocedural myocardial injury [i.e. an elevation of serum biomarkers (preferably cardiac troponins) above the $99^{\text {th }}$ percentile of upper reference limit (URL)] was detected less commonly after PCI in Ranolazine than in control group ( $11 \%$ vs. $27 \%, \mathrm{p}=0.0001)$. Also, PCI-related myocardial infarction [i.e., post procedural increase in $\mathrm{CK}-\mathrm{MB}>3$ times above the URL] tended to be lower in the Ranolazine versus placebo group: $1.8 \%$ versus $5.45 \%, \mathrm{P}=0.0002 .24$ hours post procedural levels of cardiac markers were also significantly lower in the Ranolazine versus control group (CK-MB: $2.42 \pm 2.05$ versus $7.02 \pm 9$ $\mathrm{ng} / \mathrm{ml}, \mathrm{P}=0.001$; Troponin 1: $0.447 \pm 0.74$ versus $1.18 \pm 1.6 \mathrm{ng} / \mathrm{ml}, \mathrm{P}=0.004)$. No significant adverse effect of the drug was reported. There were no inhospital and 30-day Major Adverse Cardiac Events (cardiac death, spontaneous MI, target vessel revascularization and stroke) in any of the groups.

Table-1: Preprocedural medications and baseline investigations in the two groups $(n=110)$ :

\begin{tabular}{llll}
\hline Variables Case & $(\mathbf{n =}=\mathbf{5 5})$ & Control $(\mathbf{n = 5 5})$ & p value \\
\hline Medications & & & \\
Aspirin, n(\%) & $55(100)$ & $55(100)$ & 1.000 \\
Clopidogrel loading n(\%) & $55(100)$ & $55(100)$ & 1.000 \\
Statins, $\mathrm{n}(\%)$ & $55(100)$ & $55(100)$ & 1.000 \\
Beta Blockers, n(\%) & $47(85.45)$ & $50(90.9)$ & 0.313 \\
ACEI/ARB,n(\%) & $31(56.4)$ & $30(54.5)$ & 0.967 \\
Nitrates, n(\%) & $51(92.7)$ & $53(96.4)$ & 0.687 \\
Investigations before enrollment & & \\
S. creatinine (mg/dl) & $0.91 \pm 0.3$ & $0.91 \pm 0.2$ & 0.317 \\
SGPT (IU/L) & $21 \pm 10$ & $21 \pm 19$ & 0.898 \\
WBC Total count (U/L) & $6931 \pm 1820$ & $6647 \pm 1950$ & 0.446 \\
\hline
\end{tabular}

Table-1 shows that the percentage of patients receiving Antiplatelets, statins, beta blockers, ACEI/ ARB and nitrates were similar in both the groups
Table-2: General Characteristics of the patients enrolled in the two groups $(n=110)$ :

\begin{tabular}{|c|c|c|c|}
\hline Variables & Case $(n=55)$ & Control $(n=55)$ & p value \\
\hline Age (years) & $49 \pm 5$ & $50 \pm 7$ & 0.481 \\
\hline $\begin{array}{l}\text { Male, } \mathrm{n}(\%) \\
\text { ("render }\end{array}$ & $41(74.5)$ & $43(78.2)$ & 0.447 \\
\hline Female, $\mathrm{n}(\%)$ & $15(25.5)$ & $12(21.8)$ & 0.447 \\
\hline BMI (kg/m2) & $25 \pm 2$ & $25 \pm 3$ & 0.368 \\
\hline $\begin{array}{l}\text { Hypertension, } \\
\mathrm{n}(\%)\end{array}$ & $38(69.1)$ & $40(72.7)$ & 0.812 \\
\hline $\begin{array}{l}\text { Current Smokers, } \\
\mathrm{n}(\%)\end{array}$ & $18(32.7)$ & $21(38.2)$ & 0.940 \\
\hline Diabetes, n(\%) & 17(30.9) & $16(29.1)$ & 0.972 \\
\hline $\begin{array}{l}\text { Dyslipidemia, } \\
\mathrm{n}(\%)\end{array}$ & $23(41.8)$ & $20(36.4)$ & 0.438 \\
\hline $\begin{array}{l}\text { Family History } \\
\text { of IHD, n(\%) }\end{array}$ & $7(12.7)$ & $8(14.5)$ & 0.983 \\
\hline $\begin{array}{l}\text { Previous } \\
\text { Stroke, } n(\%) j\end{array}$ & 0 & 0 & \\
\hline $\begin{array}{l}\text { Previous } \\
\text { Myocardial } \\
\text { Infarction, } \mathrm{n}(\%)\end{array}$ & 5 & 4 & 0.653 \\
\hline \multicolumn{4}{|c|}{ Previous Coronary Intervention, $\mathrm{n}(\%) 00$} \\
\hline Left Ventricular & ction Fract & (\%) $57 \pm 658=$ & 976 \\
\hline
\end{tabular}

Table-2 shows both ranolazine and control groups were similar with regard to their demographic and clinical characteristics.

Table-3: Distribution of patients based on increase in Post procedural cardiac biomarker level $(n=110)$.

\begin{tabular}{llll}
\hline CK-MB & Case(n=55) & Control(n=55) & p value \\
\hline No increase, n(\%) & $49(89.1)$ & $40(72.72)$ & 0.0002 \\
$\begin{array}{l}\text { Any elevation } \\
\text { above URL, n(\%) }\end{array}$ & $5(9.1)$ & $12(21.83)$ & 0.0002 \\
$\begin{array}{l}\text { Elevation >3-time, n(\%) } \\
\text { 24 hours after }\end{array}$ & $2.42 \pm 2.05$ & $7.02 \pm 9$ & 0.001 \\
PCI (ng/ml) & & & \\
Troponin I & & & \\
No increase, n(\%) & $48(87.27)$ & $37(67.27)$ & 0.0001 \\
$\begin{array}{l}\text { Any elevation } \\
\text { above URL, n(\%) }\end{array}$ & $6(11)$ & $15(27.27)$ & 0.0001 \\
$\begin{array}{l}\text { Elevation >3-time, n(\%) } \\
\text { 24 hours after }\end{array}$ & $1(1.8)$ & $3(5.45)$ & 0.0001 \\
PCI (ng/ml) & $0.447 \pm 0.74$ & $1.18 \pm 1.6$ & 0.004 \\
\hline Table-3 shows that & & & \\
\hline
\end{tabular}

Table-3 shows that periprocedural myocardial injury was detected in fewer patients in the Ranolazine group 
Effectiveness of Ranolazine to Prevent Myocardial Injury

than in control group. There were less patients in the Ranolazine than in control group with increases in CKMB level above the URL. Overall, PCI-related myocardial infarction occurred in fewer patients in the Ranolazine group versus the control group. Mean preprocedural levels of the 2 markers were similar and were within the normal limit in the 2 groups. However, the values obtained 24 hours post-PCI were significantly lower in Ranolazine group than those in control group.

\section{Discussion}

This study demonstrates that pretreatment with Ranolazine $1000 \mathrm{mg}$ twice daily for 7 days significantly reduced periprocedural myocardial injury (11\% versus $27.27 \%, \mathrm{P}=0.0001)$ and PCI-related myocardial infarction $(1.8 \%$ versus $5.45 \%$, $\mathrm{P}=0.0002$ ) during elective PCI the results of our investigation support the hypothesis that Ranolazine may offer protection to cardiac myocyte from ischemic injury during PCI. Ranolazine has a unique mechanism of action because it prevents the increase in intracellular calcium concentration in ischemic myocardium. Our finding is consistent with the first pilot, randomized trial ${ }^{17}$ which demonstrated that pretreatment with Ranolazine is effective in decreasing the incidence of myocardial injury during PCI. Indeed, Ranolazine significantly reduced release of all markers of myocardial damage after PCI, including CK-MB and Troponin-I. No significant side effect of Ranolazine was reported during this study and QTc was not found to be prolonged after treatment with Ranolazine. So, Ranolazine was found to be safe in this study as was found in previous studies. ${ }^{13,14,15,16}$

However, the incidence of periprocedural myocardial injury and PCI-related myocardial infarction in both groups were lower than that observed in the pilot study ${ }^{17}$. In the pilot study, PMI was observed in $23 \%$ of patients in Ranolazine group and $40 \%$ of patients in control group, whereas PCI-related myocardial infarction occurred in 6\% patients in Ranolazine group and $22 \%$ of patients in control group. This low incidence in our study can be explained by the discrepancies of several factors which have major implications in the incidence of PMI. First, the mean age of patients in our study (49 years in Ranolazine and 50 years in control group) was lower than that in the pilot study (64 years in Ranolazine and 60 years in control group). Second, the number of cases where the
LAD was intervened was lower in our study $(50 \%$ in Ranolazine and $48 \%$ in control group) compared to the previous study (69\% in Ranolazine and $57 \%$ in control group). Third, the number of stents deployed per patient was lower in this study (1.6 in Ranolazine and 1.46 in control group) than that in the pilot study (1.95 for Ranolazine and 1.75 in control group). Fourth, postdilatation was done in fewer cases in our study $(6.8 \%$ in Ranolazine and $8.4 \%$ in control group) compared to the cases in the pilot study (69\% in Ranolazine and $71 \%$ in control group). The use of fewer post-dilatation has reduced the total ischemic time and might have contributed to the lower incidence of PMI in our study. Fifth, intervention of bifurcation lesion with kissing balloon was done in the pilot study in $26 \%$ of cases in Ranolazine group and $20 \%$ cases in the control group. But, this complex technique was not applied in any patient of our study. Lastly, there might have been some ethnic and genetic variation in the susceptibility to PMI in our patients compared to the European patients. We found no major adverse cardiac event (MACE) during the in-hospital and after one month in any of the groups.

\section{Conclusion}

Percutaneous coronary intervention (PCI) is a wellestablished and relatively safe procedure for the treatment of chronic stable angina. However, periprocedural myocardial injury (PMI) is common after PCI and has an important impact on long-term prognosis. PMI complicating PCI has not received as much emphasis as other aspects of PCI. So, the patients of chronic stable angina who are scheduled for elective PCI deserve an intensive application of preventive measures to be protected from PMI. Different treatments are available to prevent myocardial injury during elective PCI. Nevertheless, the incidence of PMI is high. So, adjunctive therapy for further protection of the myocardium from ischemic insult at the time of PCI is still needed. Ranolazine is a safe \& effective oral medication which was shown to be effective in reducing PMI during elective PCI. So, this novel pharmacological agent offer hope in further reduction of the incidence of PMI.

\section{Acknowledgement}

I would like to acknowledge the enthusiastic guidance of all my teachers of Department of Cardiology, 
BSMMU, Dhaka. I express my deepest gratitude to them for their supervision, support and advice in each and every step throughout the thesis work.

Conflict of Interest: None.

\section{References}

1. World Health Organization Website http:// www. who.int. Accessed March 26, 2012.

2. Murray CJL, Lopez AD. The Global Burden of Disease: A Comprehensive Assessment of Mortality and Disability from Disease; Injuries and Risk Factors in 1990 and Projected to 2020. Boston, Ma: Harvard University Press; 1996.

3. Heron MP, Hoyret DL, Xu J, et al. Deaths: Preliminary data for 2006. Nati Vital Stat Rep. 2008; 56: 1-52.

4. Zaman MM, Ahmed J, Choudhury SR, et al. 'Prevalence of Ischemic heart disease in a rural population of Bangladesh', Indian Heart J, 2007; 59(3): 239-41.

5. Bhopal R, Unwin $\mathrm{N}$, White $\mathrm{M}$, et al. Heterogeneity of Coronary heart disease risk factors in Indian, Pakistani, Bangladeshi and European Origin Population: Cross Sectional Study. BMJ. 1999 July 24; 319: 215-220.

6. Babu GG, Walker JM, Yellon DM, et al. Periprocedural myocardial injury during percutaneous coronary intervention: an important target for cardioprotection. Eur Heart J. 2011; 32: 23-32.

7. Loannidis JP, Karvouni E, Katritsis DG. Mortality risk conferred by small elevations of creatine kinase-MB isoenzyme after percutaneous coronary intervention. $\mathrm{J} \mathrm{Am} \mathrm{Coll}$ Cardiol. 2003; 42: 1406-411.

8. Simoons ML, van den Brand M, Lincoff M, et al. Minimal myocardial damage during coronary intervention is associated with impaired outcome. Eur Heart J. 1999; 20: 1112-1119.

9. Akkerhuis KM, Alexander JH, Tardiff BE, et al. Minor myocardial damage and prognosis: are spontaneous and percutaneous coronary intervention-related events different? Circulation 2002; 105: 554-556.
10. Nienhuis MB, Ottervanger JP, Billo HJ, et al. Prognostic value of troponin after elective percutaneous coronary intervention: a metaanalysis. Catheter Cardiovasc Interv 2008; 71: 318-324.

11. Cuculi F, Lim CCS, Banning Ap. Periprocedural Myocardial injury during elective percutaneous coronary intervention: is it improtant and how can it be prevented? Heart 2010; 96: 736-740.

12. Chaitman BR. Ranolazine for the treatment of chronic Angina and potential use in other cardiovascular conditions. Circulation 2006; 113: 2462-2472.

13. Chaitman BR, Skettino SL, Parker JO, et al. for the Monotherapy Assessment of Ranolazine in Stable Angina (MARISA) trial investigators. Anti-ischemic effects and long term survival during ranolazine monotherapy in patients with chronic severe angina: a randomised controlled trial. J Am Coll Cardiol. 2004; 43: 1375-82.

14. Chaitman BR, Pepine CJ, Parker JO, et al. for the Combination Assessment of Ranolazine in Stable Angina (CARISA) trial investigators. Effects of ranolazine with atenolol, amlodipine, or diltiazem on exercise tolerance and angina frequency in patients with severe chronic angina: a randomised controlled trial. JAMA. 2004; 291: 309-16.

15. Stone PH, Gratsianky NA, Parker JO, et al., for the ERICA Investigators. Antianginal efficacy of ranolazine when added to treatment with amlodipine: the ERICA (Efficacy of Ranolazine in Chronic Angina) trial. $\mathrm{J} \mathrm{Am}$ Coll Cardiol. 2006; 48: 566-75.

16. Morrow DA, Scirica BM, KarwatowskaProkopczuk E, et al. For the MERLINTIMI 36 Trial Investigators. Effects of ranolazine on recurrent cardiovascular events in patients with non-ST-elevation acute coronary syndromes: the MERLIN-TIMI 36 randomized trial. JAMA. 2007; 297: 1775-83.

17. Pellica F, Pasceri V, Marazzi G, et al. A pilot randomized study of ranolazine for reduction of myocardial damage during elective percutaneous coronary intervention. Am Heart $J$ 2012; 163: 1019-23. 
18. Bloomfield P, Bradbury A, Grubb NR et al. Cardiovascular disease. In : Boon NA, Colledge NR, Walker BR and Hunter JA, editors. Davidson's Principles \& Practice of Medicine. $20^{\text {th }}$ edition. Elsevier limited, Philadelphia. 2010. pp. 581-591.

19. Pellica F, Pasceri V, Marazzi G, et al. Usefulness of Preprocedural Therapy with ranolazine to prevent myocardial damage in percutaneous coronary intervention. Eur Heart J. 2011; 32(Abstract Supplement): 394.

20. Chan YH. Randomized Controlled Trials (RCTs)- Sample Size: The Magic Number ? Singapore Medical J. 2003; 44(4): 172-174.

21. Abbott laboratories 2005, 'Estimation of CKMB', Operation manual for AxSYM, CK-MB AVD, Abbott laboratories, USA.

22. Abbott laboratories 2004, 'Estimation of Troponin-I, Operation manual for AxSYM, Troponin-I AVD, Abott laboratories, USA.

23. Douerman HL, Piper WD, Ahmed B. Recent Changes in the Practice of Elective PCI for Stable Angina. Circulation. 2009; 120: 8931.

24. O'Rourke RA, O'Gara PT, Shaw LJ and Douglas JS Jr. Diagnosis and Management of Patients with Chronic Ischemic Heart Disease. In Fuster V, O'Rourke RA, Walse RA, and Wilson PP editors. Hursts the Heart. 12 $2^{\text {th }}$ edition. Me Graw-Hill Companies, New York. 2008. pp. 1476-1503.
25. King SB HI, Smith SC Jr, Hirshfield JW Jr, et al. 2007 Focused Update of the ACC/AHA/SCAI 2005 Guideline Update for Percutaneous Coronary Intervention: a report of the American College of Cardiology/American Heart Association Task Force on Practice Guidelines; 2007 Writing Group to Review New Evidence and Update the ACC/AHA/SCAI 2005 Guideline Update for Percutaneous Coronary Intervention, Writing on Behalf of the 2005 Writing Committee. Circulation 2008; 117: 21- 295.

26. American Diabetes Association; Diagnosis and classification of diabetes mellitus. Diabetes Care, 2010; 33(suppl. 1)

27. Chobanian AV, Bakris GL, Black HR, et al. The Seventh Report of the National Committee on Prevention, Detection, Evaluation and Treatment of High Blood Pressure: The JNC 7 report. JAMA 2003; 289: 2560-2572.

28. US Center for Disease Control and Prevention (2010). Health behaviours of adults: United States, 2005-2007. Vital and Health Statistics, Series 10.Number 245, Appendix II, pp.80.

29. Cannon PC and Braunwald E. Unstable Angina and Non-ST-elevation Myocardial Infarction. In Libby P, Bonow RO, Mann DL, Zipes DP, editors. Heart Disease, A Text book of Cardiovascular Medicine, 8th edition. Elsevier, New Delhi. 2008. pp. 1329-1331. 